NISTIR 7482

\title{
Thermal Performance of Fire Resistive Materials II. A Multi-Layer One-Dimensional Heat Transfer Model for Fire Resistive Materials Protecting a Substrate
}

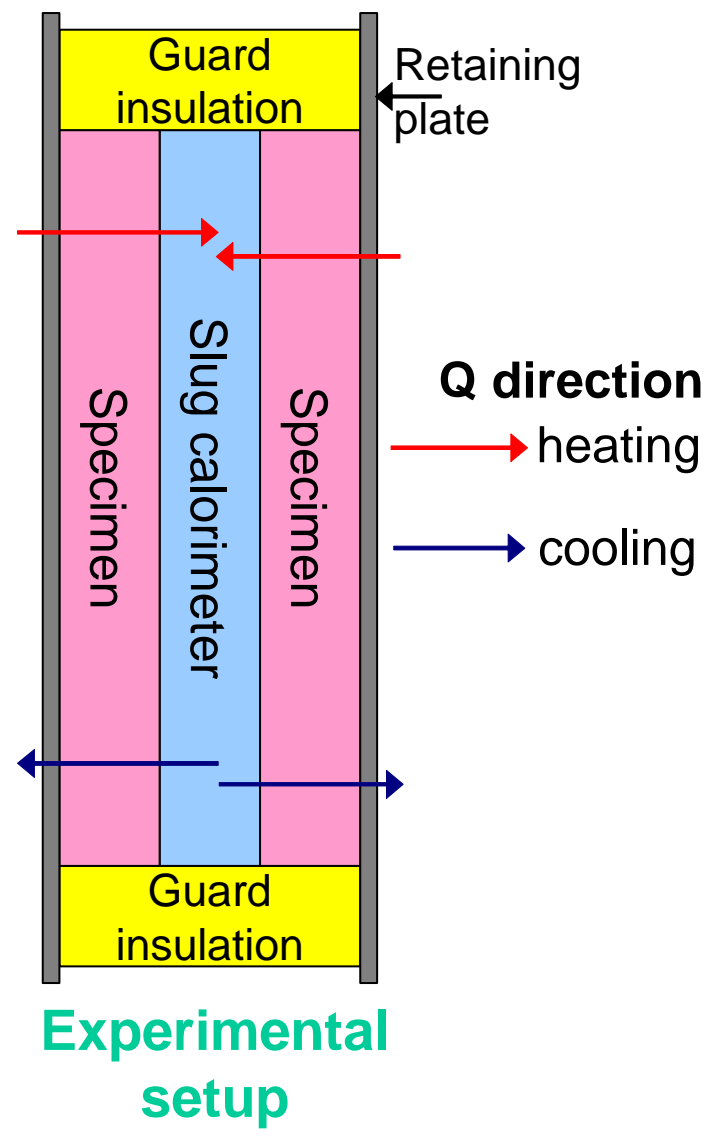

Kuldeep R. Prasad

Dale P. Bentz

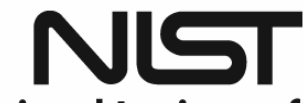

National Institute of

Standards and Technology

U.S. Department of Commerce 


\title{
Thermal Performance of Fire Resistive Materials II. A Multi-Layer One-Dimensional Heat Transfer Model for Fire Resistive Materials Protecting a Substrate
}

\author{
Kuldeep R. Prasad \\ Dale P. Bentz \\ Building and Fire Research Laboratory \\ National Institute of Standards and Technology \\ Gaithersburg, MD 20899-8615
}

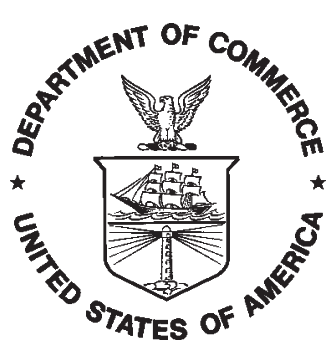

U.S. Department of Commerce Carlos M. Gutierrez, Secretary

National Institute of Standards and Technology James M. Turner, Acting Director 


\begin{abstract}
This report is the second of a three-part series concerning the characterization and modeling of the thermal performance of fire resistive materials (FRMs). These materials are currently qualified and certified based on lab-scale fire tests such as those described in the American Society for Testing and Materials (ASTM) E119 Standard Test Methods for Fire Tests of Building Construction and Materials [1]. While these tests provide an "hourly" rating for the FRM, these ratings have no direct quantitative relationship to the performance of an FRM in an actual fire, e.g., a $2 \mathrm{~h}$ rating does not mean that the FRM will protect the steel (or other substrate) for $2 \mathrm{~h}$ in a real world fire. Computational heat transfer models offer the potential to bridge the gap between laboratory testing and field performance. However, these models, whether basic one-dimensional or more complex three-dimensional versions, depend critically on having accurate values for the thermophysical properties of the FRM (and substrate) as a function of temperature, to be used as inputs along with the system geometry and fire and heat transfer boundary conditions. In part I of this series, procedures for determining a consistent set of these thermophysical properties were presented. Now, in part II, a computational onedimensional multi-layer model for the heat transfer from the fire, through the FRM, to the substrate is developed and verified by comparison to the results of a series of slug calorimeter experiments, previously conducted in the Building and Fire Research Laboratory (BFRL). Ultimately, similar performance simulations will be executed for ASTM E119-type tests and even real fires.
\end{abstract}




\section{Table of Contents}

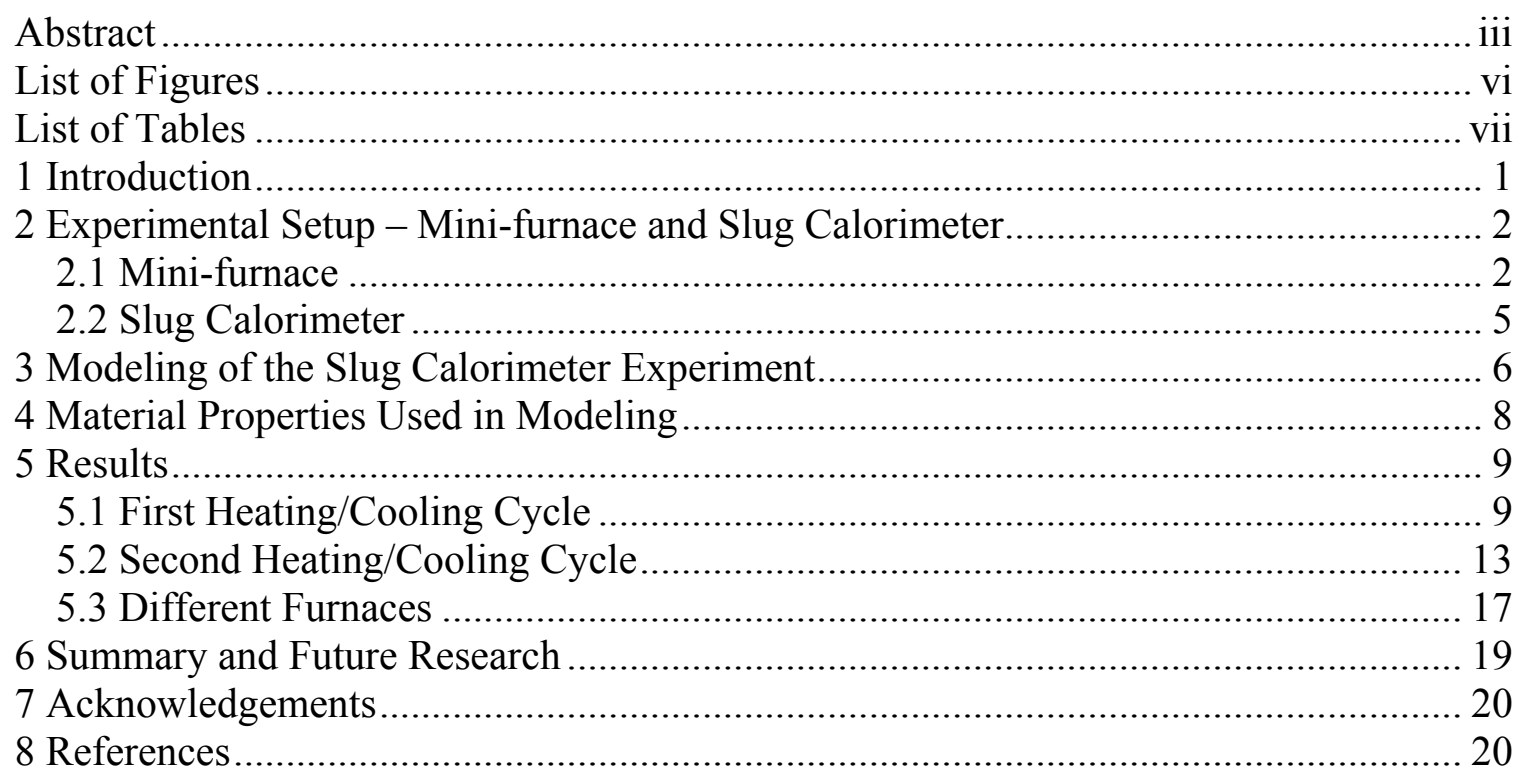




\section{List of Figures}

Figure 1. Schematics of the slug calorimeter test setup: left- schematic of a cross section through the middle of the basic slug calorimeter setup, and right- schematic of an overhead view of a completed sandwich specimen mounted and ready for testing in the mini-furnace. .

Figure 2. Comparison of measured furnace temperatures for the original furnace and the mini-furnace when programmed with the same set of linear temperature ramps. TC1 and TC2 indicate two different locations of sensing thermocouple in the minifurnace as described in the text.

Figure 3. Comparison of measured furnace and outer FRM surface temperatures for a) the original furnace and $b$ ) the mini-furnace, when programmed with the same set of linear temperature ramps

Figure 4. Experimental and model predicted exterior FRM surface (top) and slug temperatures (bottom) vs. time for the first heating/cooling cycle in the slug calorimeter for the standard density FRM.

Figure 5. Experimental and model predicted exterior FRM surface (top) and slug temperatures (bottom) vs. time for the first heating/cooling cycle in the slug calorimeter for the medium density FRM.

Figure 6. Experimental and model predicted exterior FRM surface (top) and slug temperatures (bottom) vs. time for the first heating/cooling cycle in the slug calorimeter for the high density FRM.

Figure 7. Experimental and model predicted exterior FRM surface (top) and slug temperatures (bottom) vs. time for the second heating/cooling cycle in the slug calorimeter for the standard density FRM.

Figure 8. Experimental and model predicted exterior FRM surface (top) and slug temperatures (bottom) vs. time for the second heating/cooling cycle in the slug calorimeter for the medium density FRM.

Figure 9. Experimental and model predicted exterior FRM surface (top) and slug temperatures (bottom) vs. time for the second heating/cooling cycle in the slug calorimeter for the high density FRM.

Figure 10. Experimental and model predicted exterior FRM surface (top) and slug temperatures (bottom) vs. time for the first heating/cooling cycle in the slug calorimeter in the original furnace for a standard density (specific gravity $=0.31$ ) FRM. 


\section{List of Tables}

Table 1. Specific Gravities (with standard deviations from the twin specimens) of the

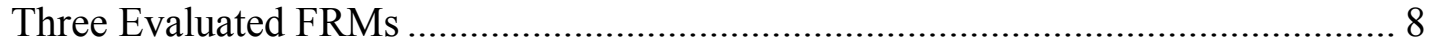




\section{Introduction}

In recent years, computer modeling has been successfully applied in bridging the gap between laboratory measurements and field performance predictions for a wide variety of materials. A prime example of this were the simulations conducted as part of the investigation of the World Trade Center progressive collapse led by the Building and Fire Research Laboratory (BFRL) of the National Institute of Standards and Technology (NIST) [2]. This current series of NIST internal reports specifically addresses the modeling of heat transfer from a fire (or furnace) through a fire resistive material (FRM) to the (steel) substrate that the FRM is protecting. The necessary characterization of the FRMs and substrate materials was outlined in part I of this series [3]. In part II, the details of a one-dimensional multi-layer heat transfer model are presented and the model is verified by comparison against a variety of slug calorimeter experiments performed previously in BFRL. In part III, extensions of the modeling to American Society for Testing and Materials (ASTM) E-119 [1] standard fire tests and real world fires will be considered. 


\section{Experimental Setup - Mini-furnace and Slug Calorimeter}

\subsection{Mini-furnace}

Originally, slug calorimeter experiments were conducted in an electrically-heated box furnace with a working volume of $360 \mathrm{~mm}$ by $360 \mathrm{~mm}$ by $360 \mathrm{~mm}$, a maximum operating temperature of $1773 \mathrm{~K}$, and heating provided from exposed heating elements on all four vertical faces of the interior $[4,5]$. However, it soon became desirable to demonstrate that equivalent measurements could be achieved using a smaller and less expensive furnace. The mini-furnace constructed to meet this objective has a working volume of $250 \mathrm{~mm}$ by $250 \mathrm{~mm}$ by $300 \mathrm{~mm}$ and is constructed from a set of four ceramic fiber elements. As shown in Figure 1, only two of the elements (sides) contain active heating, while the other two elements (front and back) function as passive insulators. The active heating elements measure $356 \mathrm{~mm} \times 305 \mathrm{~mm} \times 50 \mathrm{~mm}$, while the passive elements are $254 \mathrm{~mm} \times 305 \mathrm{~mm} \times 50 \mathrm{~mm}$. The top and bottom of the furnace consist of $50 \mathrm{~mm}$ thick "plates" of the same high temperature insulation that is utilized as the guard insulation in the slug calorimeter setup $[4,5]$. This mini-furnace has been used extensively and reliably for more than one year. The two active heating elements are connected to a control panel from which the temperature of the furnace can be programmed as a series of linear ramps, for example. Type $\mathrm{N}$ thermocouples, used to monitor the temperatures of the outer FRM surface, the steel slug, and the furnace, are connected to a simple Universal Serial Bus (USB)-based data acquisition unit (with cold junction compensation) that can monitor up to eight channels simultaneously, and conveniently outputs the values into a spreadsheet. The uncertainty of the Type $\mathrm{N}$ thermocouples has been presented previously [4]. For example, measurement in ice water yielded an average standard deviation of $0.05 \mathrm{~K}$ among a set of five thermocouples. At higher temperatures, the uncertainty increases to $1 \mathrm{~K}$ [4]. Recently, the slug calorimeter experimental method developed in BFRL has been standardized by ASTM as ASTM E2584 "Standard Practice for Thermal Conductivity of Materials Using a Thermal Capacitance (Slug) Calorimeter" [6].

Several comparisons were made between the original and mini furnaces to ensure compatible performance. Figure 2 shows the furnace temperatures achieved during a single heating/cooling cycle when both furnaces were programmed with the same set of piecewise linear temperature ramps for the heating portion, followed by natural cooling. Specifically, the furnace setpoints were $538{ }^{\circ} \mathrm{C}$ after $45 \mathrm{~min}, 704{ }^{\circ} \mathrm{C}$ after $70 \mathrm{~min}, 843{ }^{\circ} \mathrm{C}$ after $90 \mathrm{~min}, 927^{\circ} \mathrm{C}$ after $105 \mathrm{~min}$, and $1010^{\circ} \mathrm{C}$ after $2 \mathrm{~h}$. At the maximum points in the temperature/time curves shown in Figure 2 , the furnaces were turned off and the temperatures continually monitored during natural cooling. For the mini-furnace, results are shown for two different locations of the measurement thermocouple (37.5 $\mathrm{mm}$ and $87.5 \mathrm{~mm}$ from the heating element surface) while the thermocouple for temperature control was maintained at a distance of $37.5 \mathrm{~mm}$ in both cases, indicating the uniformity of the temperature distribution within the mini-furnace. Due to its larger working volume, the original furnace exhibits a slight lag behind the temperature rise observed in the mini-furnace. Because the two furnaces are in different local environments, the mini-furnace being housed in a fume hood and the original furnace in a large open-bay laboratory, their cooling responses are also different. But, for all practical purposes, it appears that either furnace 
may be utilized to produce a heating/cooling curve that matches the temperature setpoints presented above.
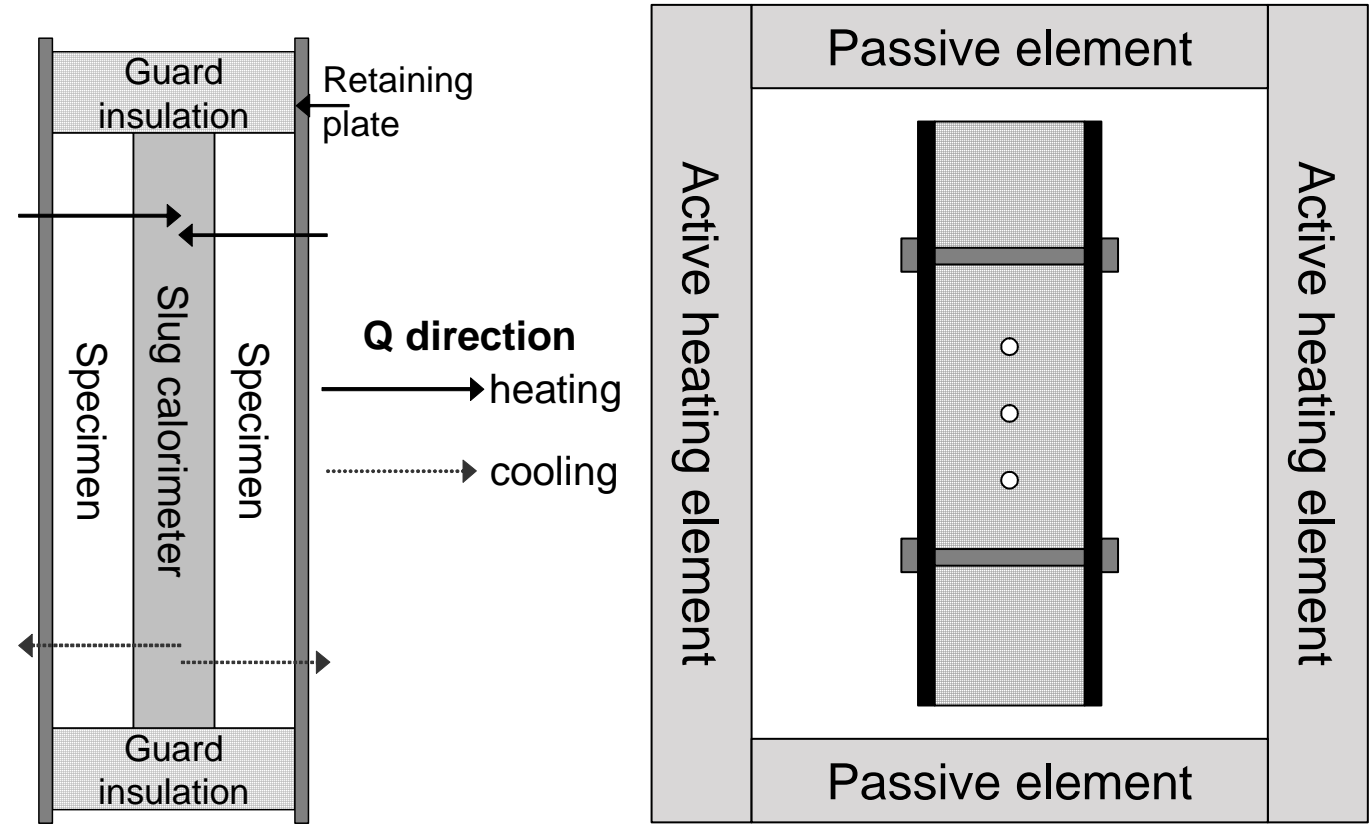

Figure 1. Schematics of the slug calorimeter test setup: left- schematic of a cross section through the middle of the basic slug calorimeter setup, and right- schematic of an overhead view of a completed sandwich specimen mounted and ready for testing in the mini-furnace.

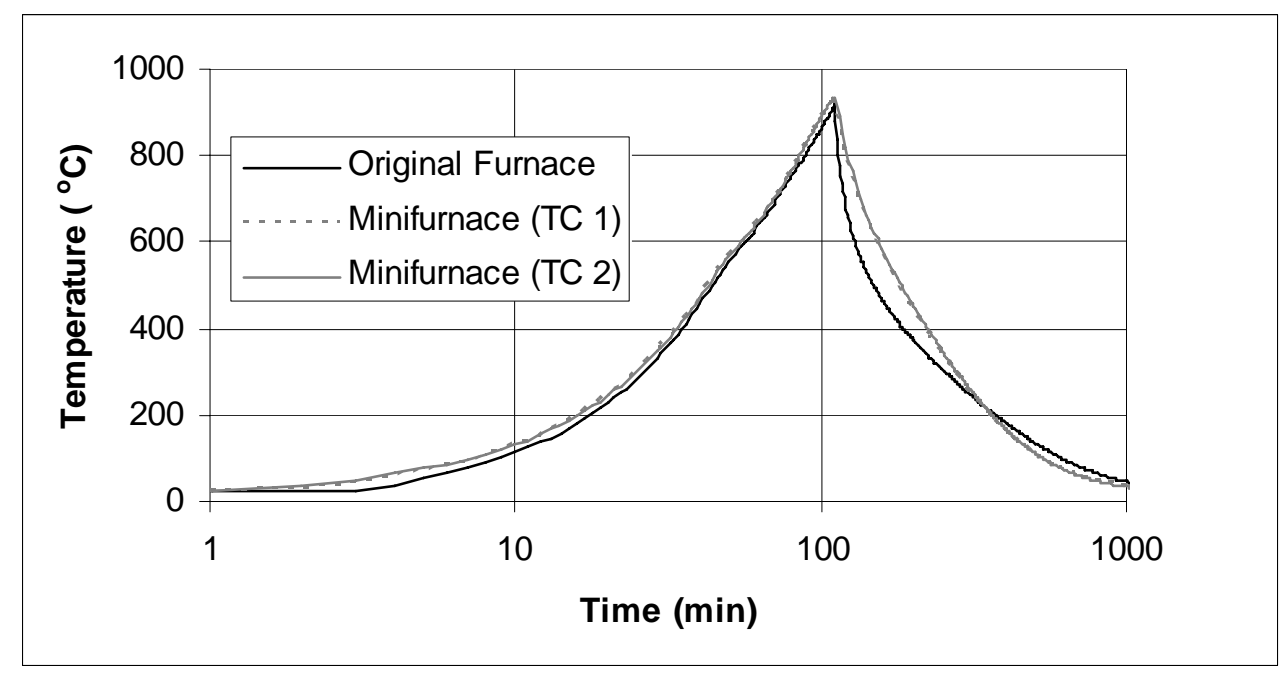

Figure 2. Comparison of measured furnace temperatures for the original furnace and the mini-furnace when programmed with the same set of linear temperature ramps. TC1 and TC2 indicate two different locations of sensing thermocouple in the mini-furnace as described in the text.

One other difference between the two furnaces was noted during the course of these experiments. Because the heating elements in the original furnace are exposed, they will transfer considerable energy via radiation to the Inconel retaining plates located on the exterior of the slug calorimeter setup (Figure 1). Thus, at the later stages of the heating curve, as shown in 
Figure 3, the temperature of the outer surfaces of the FRM specimens (and these retaining plates) actually may exceed that of the furnace environment. For the mini-furnace, where the heating elements are embedded in the ceramic fiber boards, this (radiation) effect is not observed, as the temperature of the outer FRM surface always remains below that of the furnace environment during heating (Figure 3). From a practical viewpoint, either of these cases is reasonable, as long as the temperatures of the steel slug and outer FRM surface are monitored throughout the heating/cooling cycles. However, as will be shown later, from a modeling viewpoint, the covered elements of the mini-furnace are preferable, as the enhanced radiation between the open elements and the retaining plates is not easily incorporated into a model, where it is generally expected that during heating, the furnace temperature will always be higher than the outer FRM specimen temperature.
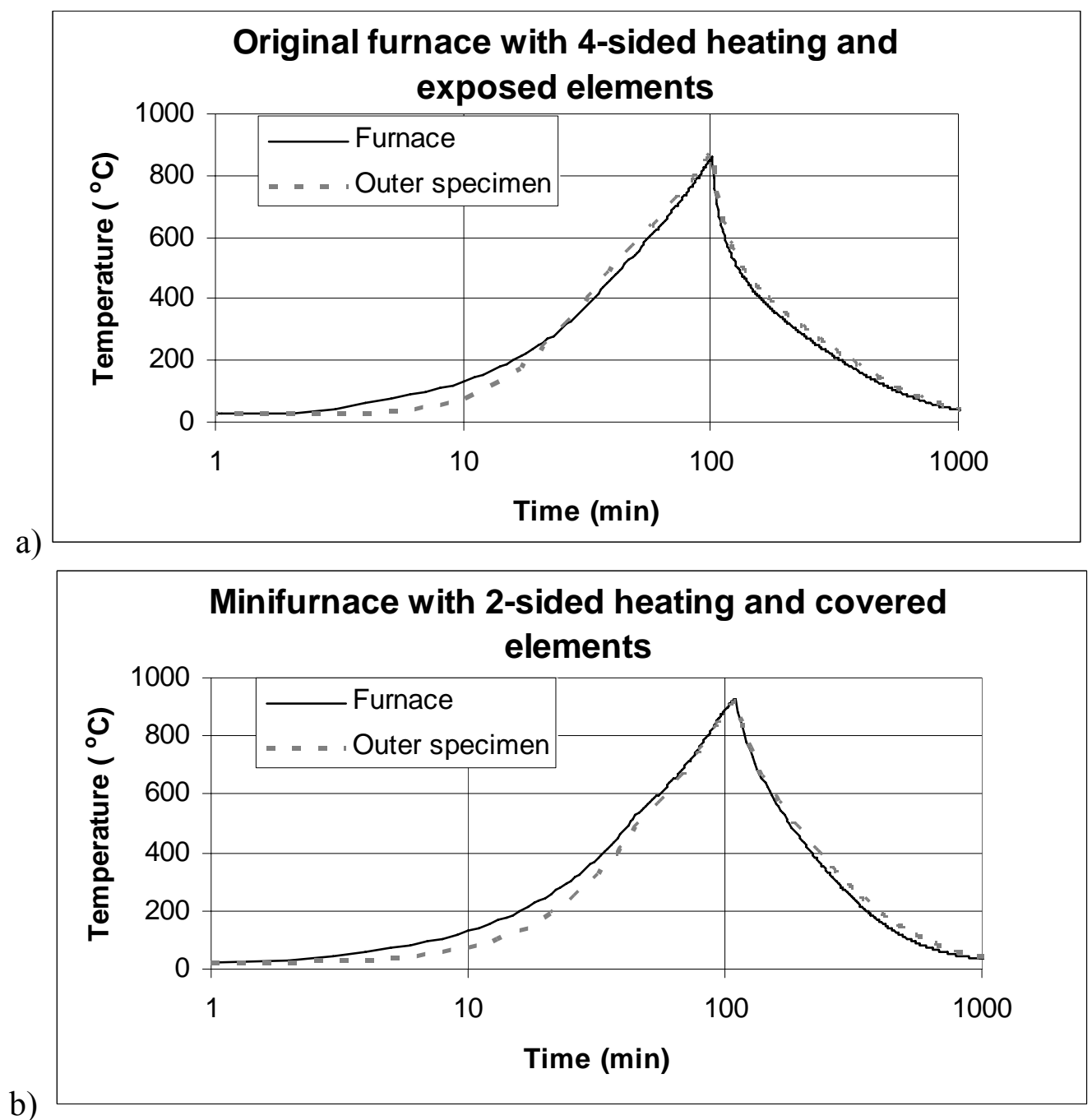

Figure 3. Comparison of measured furnace and outer FRM surface temperatures for a) the original furnace and $b)$ the mini-furnace, when programmed with the same set of linear temperature ramps. 


\subsection{Slug Calorimeter}

The details of the slug calorimeter experimental setup have been provided in references [4] and [5]. The steel slug consists of a $152.4 \mathrm{~mm}$ by $152.4 \mathrm{~mm}$ by $12.7 \mathrm{~mm}$ AISI 304 stainless steel plate into which three thermocouple holes have been milled. It has a mass of $2340 \mathrm{~g}$. The two major faces of the slug are covered with samples of the FRM to be evaluated, each nominally $25 \mathrm{~mm}$ thick. The remaining sides of the stainless steel slug (and those of the samples) are surrounded by a high temperature guard (microporous) insulation material $[4,5]$ (Figure 1). The assembled specimen is held together by two high temperature retaining plates and a set of eight bolts that are external to (outside of) the guard insulation. For the results presented in this report, the retaining plates were continuous, but contained a set of four centralized slots $(2.4 \mathrm{~mm}$ by $98 \mathrm{~mm})$ to allow for the escape of steam and gases. The calorimeter can also be assembled with retaining plates containing a $150 \mathrm{~mm}$ by $150 \mathrm{~mm}$ central square hole for when intumescent or other expansive FRMs are to be evaluated.

When ready for testing, the specimen is placed in a high temperature furnace and exposed to programmed heating/cooling cycles. Generally, the heating cycle is programmed as a series of linear ramps (such as the one provided in the previous section) and the cooling is natural (e.g., not imposed). The temperatures of the slug and the sample (exposed) surfaces are monitored and recorded over time. From this temperature/time data, the apparent thermal conductivity of the samples as a function of mean sample temperature during multiple heating/cooling cycles can be obtained from a previously derived analytical solution [4-6]. 


\section{Modeling of the Slug Calorimeter Experiment}

A numerical tool was developed to model the slug calorimeter experimental setup. As discussed in the previous section, the experimental setup consists of a slug calorimeter placed inside a furnace and subjected to a prescribed heating and cooling cycle. The slug consists of a $12.7 \mathrm{~mm}$ thick stainless steel plate covered with a layer of $25 \mathrm{~mm}$ thick FRM on its two major faces, and is protected with a high temperature guard insulation material on all the remaining faces. The assembled specimen is held together by two high temperature retaining plates. The temperature of the steel slug is measured during the course of the experiments and is a function of the thickness and thermo-physical properties of the FRM as well as the imposed heating and cooling cycle (instantaneous furnace temperature). Before proceeding to formulate the present problem of heat and moisture transfer including chemical reactions and phase changes, several important assumptions can be made to simplify the formulation. These assumptions are discussed in the next three paragraphs.

When the slug assembly is placed inside a furnace, the exterior faces of the assembly are subjected to convective and radiative heating from the furnace walls. Energy can conduct through the retaining plates and the guard insulation material. Since the thermal conductivity of the retaining plates was significantly higher than that of the guard insulation material, it can be assumed that heating of the FRM material and steel slug effectively occurs entirely through energy that was conducted through the metal retaining plates. The slug experiment can therefore be modeled simply as a one dimensional transport of mass and energy through multiple material layers.

As the FRM is heated for the first time, the material can undergo (generally) endothermic phase changes / chemical reactions. Evaporation of free water and de-hydration reactions in the FRM can result in the production of free water vapor. This free water vapor could in turn migrate (diffuse / convect) through the porous fire resistive material. It is conceivable that this free water vapor can condense in the cooler portions of the slug setup. Modeling water-vapor migration through porous materials is a difficult and challenging problem that is beyond the scope of the present report. In the current work, we will account only for the energy associated with the phase changes / chemical reactions and neglect the subsequent mass transport of water vapor through the FRM.

A further simplification can be based on symmetry arguments, noting that the slug is placed symmetrically in the furnace, the thickness of the FRM is approximately equal on both sides, and the incident fluxes on both sides are also identical. Use of the symmetry argument reduces the cost of the computations by reducing the size of the computational domain.

Consider a small volume element of a slab of homogeneous material of unit area A and of thickness $\Delta x$. The equation for conservation of energy for the control volume can be written as

$$
\frac{\partial(\rho h)}{\partial t}=-\frac{\partial}{\partial x}\left(-k \frac{\partial T}{\partial x}\right)-\frac{\partial q_{R}}{\partial x},
$$


where $\rho$ is the density of the control volume, $h$ is the enthalpy, T is the local temperature and $q_{R}$ is the radiative flux. The left hand side of the energy equation is the rate of accumulation of enthalpy in the control volume. The first term on the right hand side shows the net rate of heat addition due to thermal conduction into the control volume, while the second term on the right hand side represents the net rate of heat added due to the incident radiative flux on the control volume. The equations states that the net enthalpy change of the material in a control volume is equal to the net amount of heat entering by conduction plus the contribution due to radiative heating of the volume.

The partial differential equation expressing conservation of energy was discretized using a control volume approach to derive the finite difference form of the equations. The method of discretization ensures local energy conservation and requires approximation of only first order derivatives (rather than higher order derivatives). The goal of the numerical procedure was to predict the temperature $\mathrm{T}$ at the cell center. The discretized form of the above equation was solved for each material layer. A second order explicit Runge-Kutta scheme was used for time stepping. Although the stability characteristics of these methods are more restrictive than implicit methods, explicit Runge-Kutta techniques [7] result in more accurate solutions than implicit schemes of comparable order.

A unique solution to the governing equations requires the specification of initial and boundary conditions. The initial temperature distribution in the layers (retaining plate, FRM and stainless steel slug) was specified to match that of each experiment being modeled. The density and thermal conductivities for each material must also be defined and may or may not be a function of temperature. The enthalpy for each material must be defined as a function of temperature and can be obtained by integrating the specific heat curve and including the heat of reaction [3]. The incident radiative flux varying as a function of time must also be specified to start the calculation. Boundary conditions were also specified at the edges of the computational domain. At the gas-solid interface, the conductive heat flux into the retaining plate must equal the net radiative and convective flux reaching the surface. At the symmetry (middle) boundary, the energy flux was set to zero (adiabatic boundary condition). The procedure described in this section has been previously used for modeling the thermal performance of fire fighters protective clothing and has been discussed in detail in reference [7].

Computational grids should be constructed so as to concentrate the maximum number of grid points in the FRM layer, since it is the material of lowest thermal conductivity and also the only reactive material in the simulation. Grid density in the stainless steel slug and the retaining plate can be relatively coarse due to high thermal conductivities and specific heats for these materials. The numerical procedure chooses an appropriate time step for the time marching procedure depending on grid density, temperature and other thermo-physical properties. Typical computations were performed with approximately five grid points each in the steel plate and the retaining plate and twenty grid points in the FRM. A grid convergence study was conducted by doubling the number of grid points to make sure that the solutions were grid-independent. A typical computational cost was approximately 90 seconds on a single $3.8 \mathrm{GHz}$ processor running Linux. 


\section{Material Properties Used in Modeling}

Each FRM being evaluated using the model was characterized with respect to its density, heat capacity, apparent thermal conductivity, and enthalpies of (projected) reactions according to the methodology outlined in part I of this series [3]. In that report, example properties of five different FRMs and two types of steel, including the AISI 304 stainless steel plate used for the slug calorimeter, were provided. Based on the measured mass loss for each FRM during a high temperature (slug calorimeter) exposure, its local density was varied during the first heating cycle as a linear function of the local FRM temperature. Each FRM was represented as two separate materials in the program database, an original material (whose density was a function of temperature and whose enthalpy included reactions and phase changes) and a "burnt" material of constant (burnt) density and heat capacity, with no further reaction or phase change contributions to the enthalpy. In the program, a conversion was made for each FRM, from its "original" properties to its "burnt" properties, when the maximum furnace temperature was achieved during the first heating/cooling cycle. For any simulations of the second heating/cooling cycle, the "burnt" material properties were employed.

To illustrate the predictive capability of the model for a variety of FRM materials, standard, medium and high density products were tested in the slug calorimeter and modeled using the software described previously. One of the tested products was gypsum-based, while the other two were based on a (portland) cementitious binder. Table 1 provides the specific gravities of these three materials. Several other FRMs have also been successfully modeled using this approach, but the focus of this report will be on these three representative products.

Table 1. Specific Gravities (with standard deviations from the twin specimens) of the Three Evaluated FRMs

\begin{tabular}{|c|c|}
\hline Product & Original Specific Gravity \\
\hline Standard density & $0.36 \pm 0.01$ \\
\hline Medium density & $0.45 \pm 0.01$ \\
\hline High density & $0.77 \pm 0.01$ \\
\hline
\end{tabular}




\section{Results}

\subsection{First Heating/Cooling Cycle}

The experimental results and model predictions for the first heating/cooling cycle are compared in Figures 4, 5, and 6 for FRMs of standard, medium, and high density, respectively. In each figure, separate plots are included for the FRM (exterior) surface temperature and the slug temperature. It is observed that the FRM surface temperature predictions are in excellent agreement with the experimental values for the full heating/cooling curves. Specifically, in Figures 4,5 , and 6 , the differences in peak surface temperatures between experiment and model are $2.8^{\circ} \mathrm{C}, 9.9^{\circ} \mathrm{C}$, and $10.9^{\circ} \mathrm{C}$, respectively. This agreement suggests that an appropriate choice has been made for the heat transfer coefficient governing energy transfer between the furnace environment and the retaining plates. Because the retaining plates have a high thermal conductivity relative to any of the FRMs, the exterior FRM surface temperature is nearly identical to that of the retaining plates in the simulations conducted in this study.

Regarding the slug temperature predictions, as shown in Figures 4 through 6 , an acceptable prediction is provided by the model, particularly for temperatures exhibited during the cooling portion of the heating/cooling curves. For two of the three materials presented in these figures, the peak temperature of the slug is slightly underpredicted by the modeling. Specifically, in Figures 4, 5, and 6, the differences in peak slug temperatures between experiment and model are $29.1{ }^{\circ} \mathrm{C}, 1.4{ }^{\circ} \mathrm{C}$, and $23.7^{\circ} \mathrm{C}$, respectively. In addition, for each material, the agreement between model and experiment for slug temperatures less than $100{ }^{\circ} \mathrm{C}$ during heating is not particularly good. Initially, the measured slug temperature is significantly higher than the predicted one, followed by a slight plateau region in the measured results, when the slug achieves a temperature of about $100{ }^{\circ} \mathrm{C}$. No such plateau is produced by the modeling. It is hypothesized that both the difference between measured and model temperatures at these lower temperatures and the presence of this plateau in the experimental results are due to mass transport and subsequent evaporation (boiling) of condensed moisture released as the gypsumbased or cementitious binders of the different FRMs dehydrate (only) during the first heating cycle in the furnace. As this dehydration front penetrates into the FRM from the exterior surface, steam is driven towards the interior of the FRM specimen (and the slug surface), where it may first recondense when it reaches a local environment with a temperature below $100{ }^{\circ} \mathrm{C}$ and then evaporates as that local temperature is raised above $100{ }^{\circ} \mathrm{C}$ due to heat transfer from the furnace (or fire) through the FRM. The initial mass transfer of the steam/hot water results in experimental slug temperatures above those predicted by the model, while its ultimate evaporation absorbs energy (at the slug surface), such that the temperature rise of the slug in the experiment plateaus near $100{ }^{\circ} \mathrm{C}$ and is less than that predicted by the model. The net effect, however, appears to be fairly well accounted for in the model by including the enthalpies of dehydration (and decarbonation) of the binder components of the various FRM materials. The hypothesis that these differences are due to dehydration reactions and mass transport is further supported by the fact that these effects are not observed at all during the second heating/cooling cycle (Figures 7 through 9), as the FRM has completely reacted and all moisture has been completely removed during the first heating/cooling cycle. 

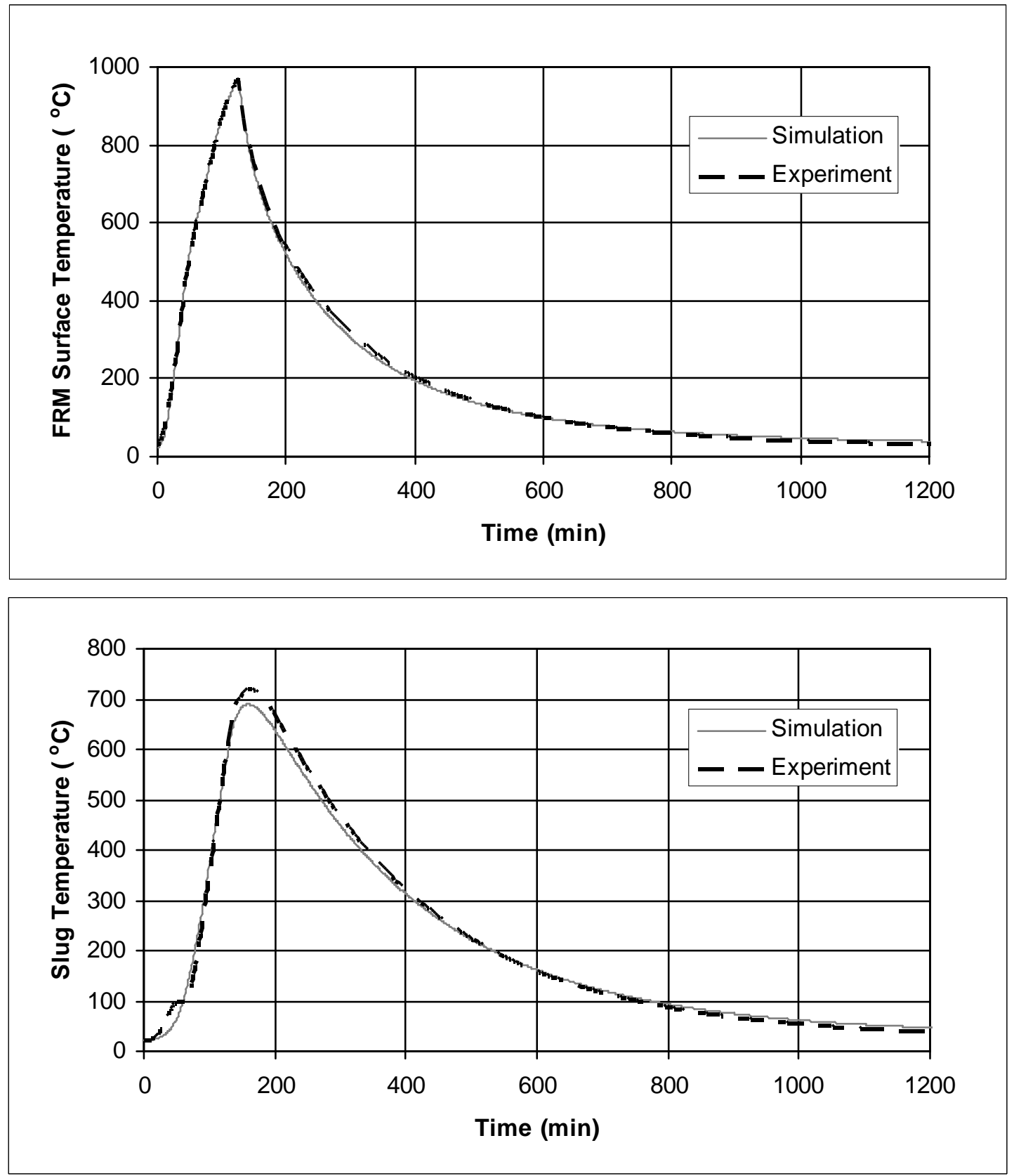

Figure 4. Experimental and model predicted exterior FRM surface (top) and slug temperatures (bottom) vs. time for the first heating/cooling cycle in the slug calorimeter for the standard density FRM. 

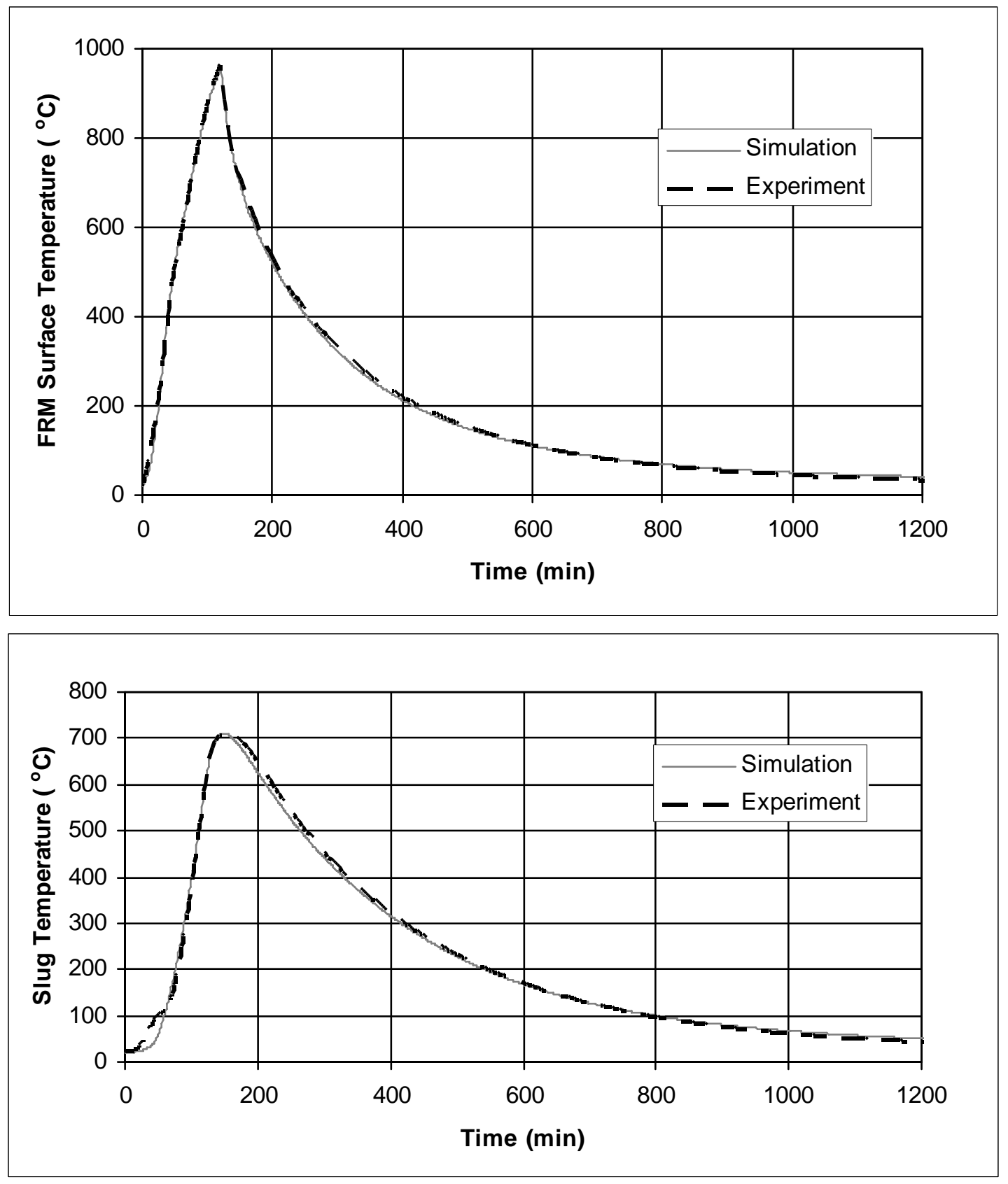

Figure 5. Experimental and model predicted exterior FRM surface (top) and slug temperatures (bottom) vs. time for the first heating/cooling cycle in the slug calorimeter for the medium density FRM. 

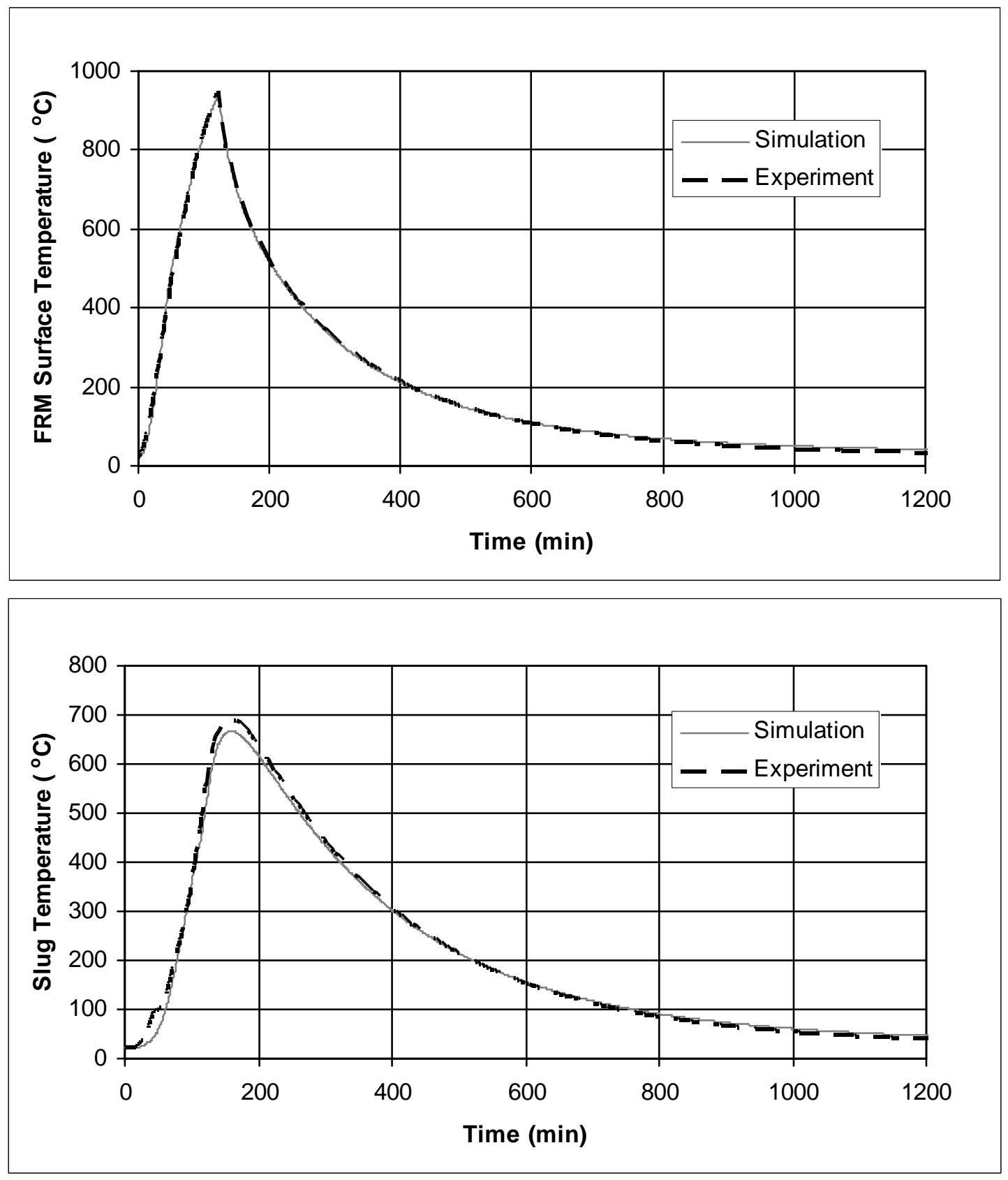

Figure 6. Experimental and model predicted exterior FRM surface (top) and slug temperatures (bottom) vs. time for the first heating/cooling cycle in the slug calorimeter for the high density FRM. 


\subsection{Second Heating/Cooling Cycle}

The experimental results and model predictions for the second heating/cooling cycle are compared in Figures 7, 8, and 9 for FRMs of standard, medium, and high density, respectively. In each case, excellent agreement is observed between the model and experimental results for both the FRM surface and the stainless steel slug temperatures. For these simulations, the differences in peak surface temperatures between experiment and model are $3.0^{\circ} \mathrm{C}, 12.5^{\circ} \mathrm{C}$, and $7.8^{\circ} \mathrm{C}$, for the standard, medium, and high density FRMs, respectively. For the peak slug temperatures, the corresponding differences are $6.3{ }^{\circ} \mathrm{C}, 1.2{ }^{\circ} \mathrm{C}$, and $4.6{ }^{\circ} \mathrm{C}$, respectively. During the $2^{\text {nd }}$ heating curve, no indication of a temperature plateau near $100^{\circ} \mathrm{C}$ is observed, suggesting that all dehydration reactions went to completion during the first heating cycle and that all of the free and bound water was effectively removed from the FRM (and the slug calorimeter setup) during the first heating/cooling cycle, as noted previously. 

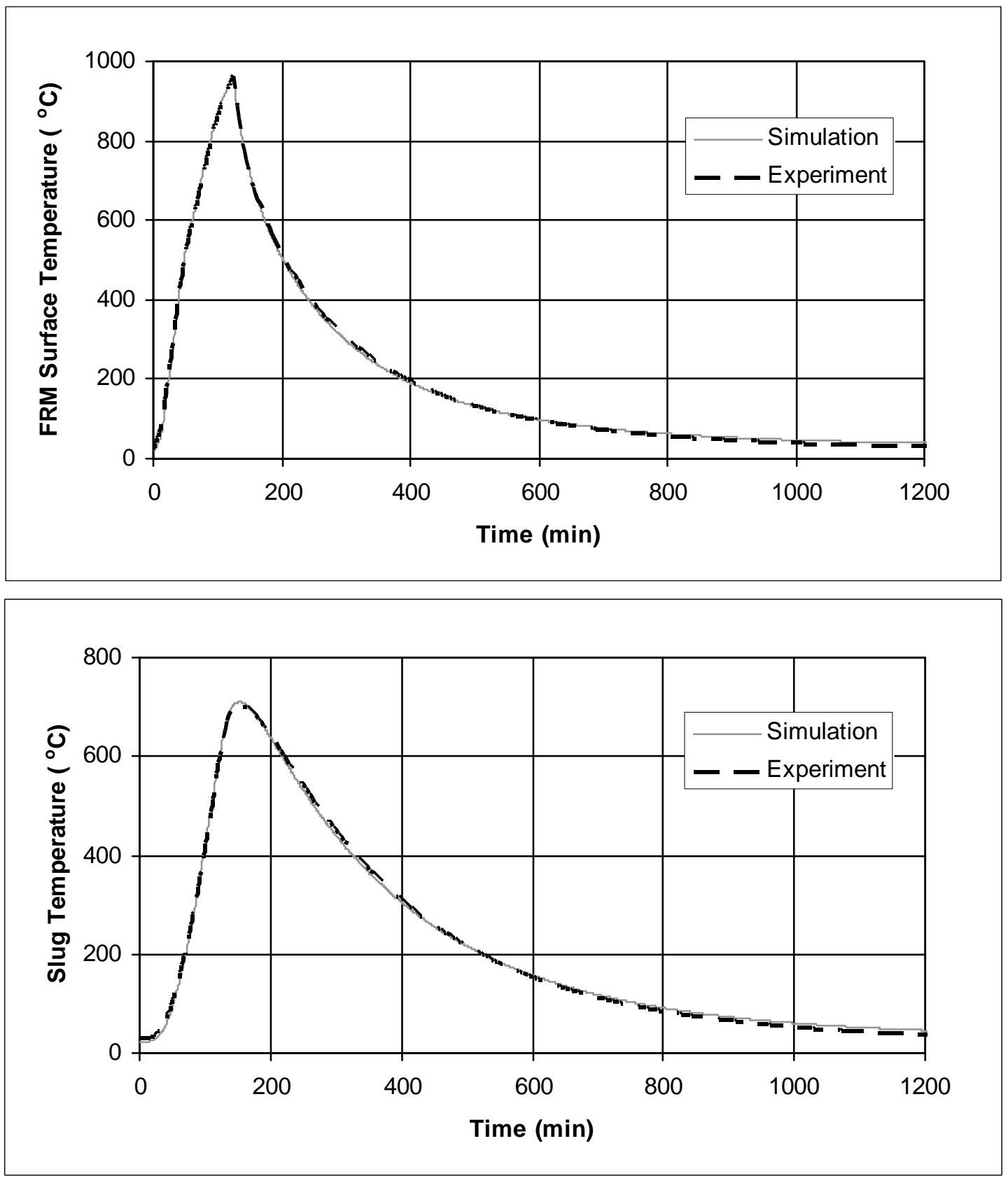

Figure 7. Experimental and model predicted exterior FRM surface (top) and slug temperatures (bottom) vs. time for the second heating/cooling cycle in the slug calorimeter for the standard density FRM. 

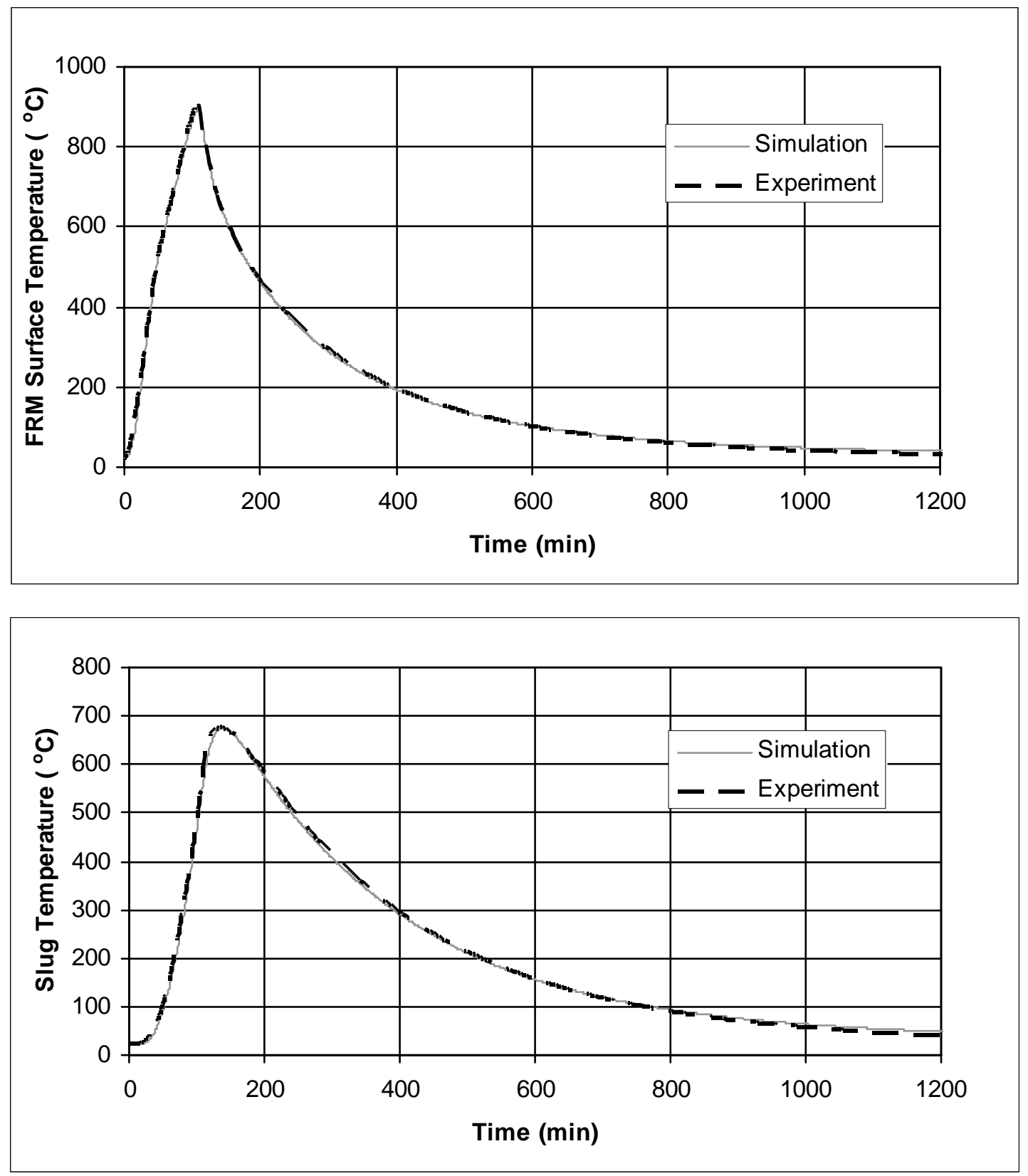

Figure 8. Experimental and model predicted exterior FRM surface (top) and slug temperatures (bottom) vs. time for the second heating/cooling cycle in the slug calorimeter for the medium density FRM. 

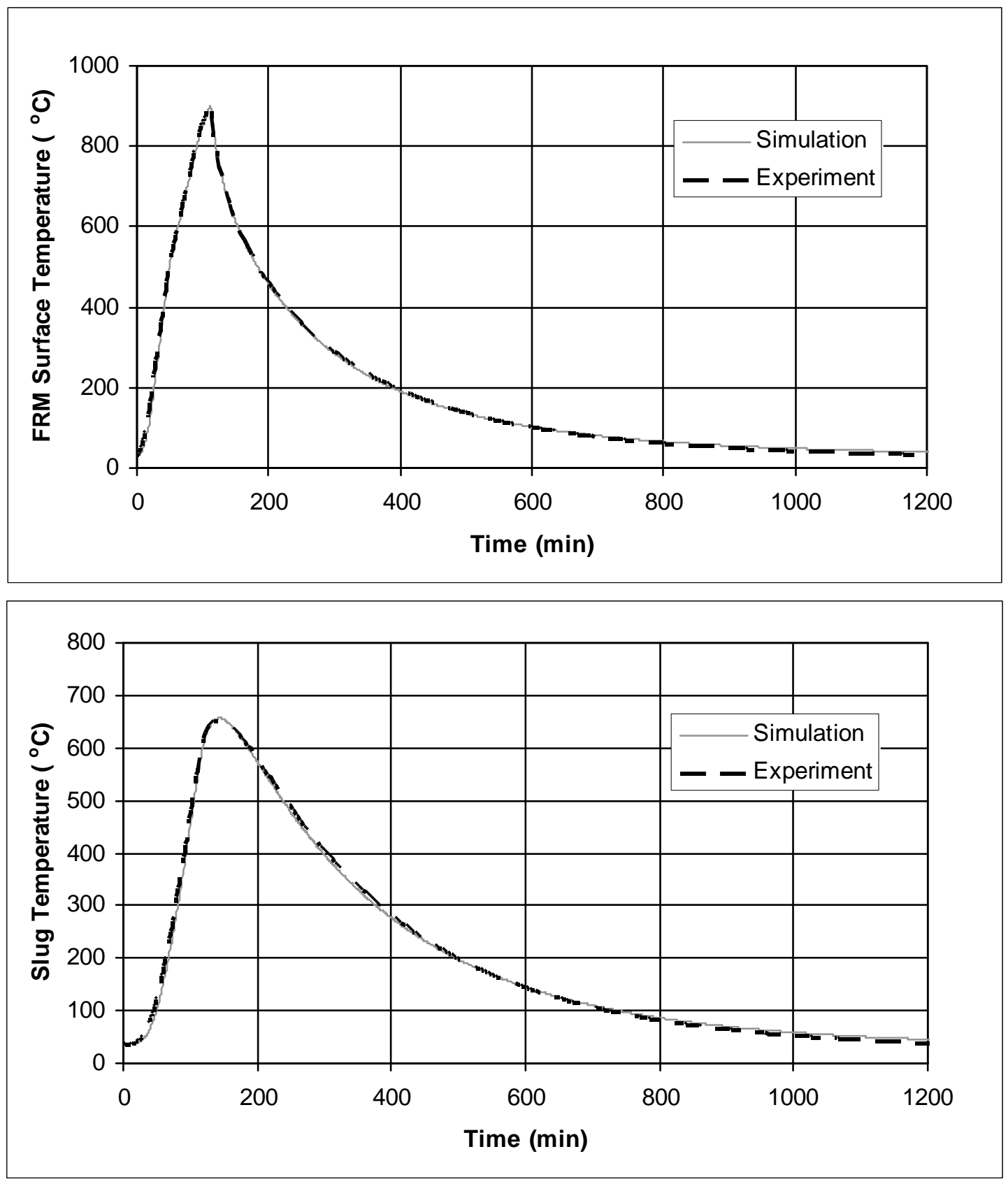

Figure 9. Experimental and model predicted exterior FRM surface (top) and slug temperatures (bottom) vs. time for the second heating/cooling cycle in the slug calorimeter for the high density FRM. 


\subsection{Different Furnaces}

As was mentioned previously, the open heating elements present in the original furnace result in a different energy transfer between the furnace "walls" and the slug calorimeter's retaining plates. The additional radiative transfer between the glowing elements and the metal plates complicates any modeling of the experimental results, as shown in Figure 10. For this furnace, the simulation results consistently underestimate both the experimental exterior FRM surface and slug temperatures for much of the heating portion of the heating/cooling cycle. Specifically, the maximum surface and slug temperatures are underestimated by $31{ }^{\circ} \mathrm{C}$ and $72{ }^{\circ} \mathrm{C}$, respectively. While the heat transfer coefficient between the furnace and the retaining plates could be adjusted (increased) to improve the agreement between experiment and simulation, the increase of the retaining plate (FRM surface) temperature above that of the furnace will not be obtained in the current configuration of the model, regardless of what value is employed for the heat transfer coefficient. An extended model that accounted for the energy transfer between the individual heating elements and the metal retaining plates would be required when the heating elements are directly exposed. Fortunately, for the insulated heating elements employed in the mini-furnace, it appears that such energy transfer is negligible and thus, the slug calorimeter experiment can be successfully modeled using the one-dimensional multi-layer heat transfer model presented in this report. 

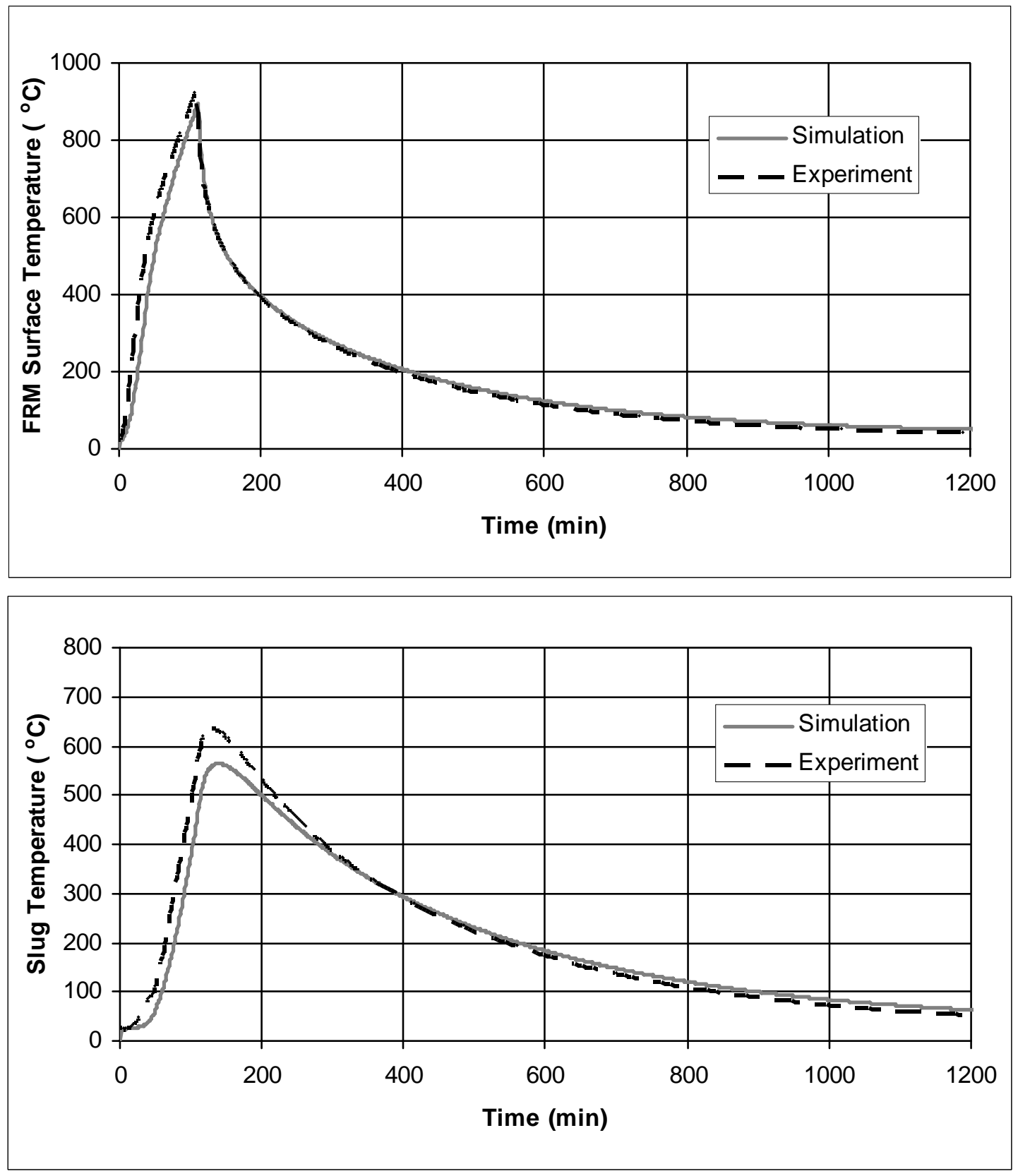

Figure 10. Experimental and model predicted exterior FRM surface (top) and slug temperatures (bottom) vs. time for the first heating/cooling cycle in the slug calorimeter in the original furnace for a standard density (specific gravity $=0.31$ ) FRM. 


\section{Summary and Future Research}

This report has demonstrated the capability of a multi-layer thermal performance model to simulate the results of a slug calorimeter experiment exposure in a high temperature furnace. By including the enthalpy of the FRM as a function of temperature, along with its thermal conductivity and mass loss, adequate predictions of the stainless steel slug temperature during both the first and second heating/cooling cycles could be produced for a furnace exposure where the heating elements were encased within the furnace walls. Conversely, results for a furnace with exposed elements differed significantly from the experimental measurements, most likely due to enhanced radiative transfer between these elements and the metal retaining plates used to immobilize the slug calorimeter specimen. Future studies will extend the modeling to consider actual ASTM E119 fire exposures, beginning with testing of structural steel columns protected by an FRM. If these efforts are successful, it is envisioned that the model will be incorporated into a computational toolkit to be made available to the general public from NIST. Further refinements of the model could also be considered, such as including a mass transfer component to simulate the moisture movement that occurs during the first heating cycle of the slug calorimeter. To accurately model this phenomena, in addition to the moisture release rates (dehydration kinetics), gas and liquid permeabilities of the FRM as a function of temperature would also be needed. The initial one-dimensional heat transfer model for firefighter's clothing [7] already includes the appropriate basic equations to simulate such moisture movement. 


\section{Acknowledgements}

The authors would like to acknowledge the financial and technical support of the current industrial members of the National Institute of Standards and Technology/industry consortium on Performance Assessment and Optimization of Fire Resistive Materials: the American Iron and Steel Institute, Isolatek International, PPG Industries, and W.R. Grace \& Co.- Conn.

\section{References}

[1] ASTM E119, "Standard Test Methods for Fire Tests of Building Construction and Materials," ASTM Annual Book of Standards, ASTM International, West Conshohocken 2007.

[2] NIST NCSTAR 1: Federal Building and Fire Safety Investigation of the World Trade Center Disaster: Final Report on the Collapse of the World Trade Center Towers, U.S. Department of Commerce, September 2005.

[3] Bentz, D.P., Prasad, K.R., "Thermal Performance of Fire Resistive Materials I. Characterization of Fire Resistive Materials with Respect to Thermal Performance Models," NISTIR 7401, U.S. Department of Commerce, February 2007.

[4] Bentz, D.P., Flynn, D.R., Kim, J.H., and Zarr, R.R., "A Slug Calorimeter for Evaluating the Thermal Performance of Fire Resistive Materials," Fire and Materials, Vol. 30 (4), 257-270 (2006).

[5] Bentz, D.P., "Combination of Transient Plane Source and Slug Calorimeter Measurements to Estimate the Thermal Properties of Fire Resistive Materials," Journal of Testing and Evaluation, Vol. 35 (3), 240-244, (2007).

[6] ASTM E2584, "Standard Practice for Thermal Conductivity of Materials Using a Thermal Capacitance (Slug) Calorimeter," ASTM Annual Book of Standards, ASTM International, West Conshohocken 2007.

[7] Prasad, K., Twilley, W., and Lawson, J.R., "Thermal Performance of Fire Fighters' Protective Clothing. 1. Numerical Study of Transient Heat and Water Vapor Transfer," NISTIR 6881, U.S. Department of Commerce, August 2002. 